\title{
Different Zoom Approaches for Improving Spectral Resolution with Applications in Radar Signal Processing
}

\author{
A. Hossen*a and U. Heute ${ }^{\mathrm{b}}$ \\ *a Department of Electrical and Computer Engineering, PO Box 33, PC 123, Al-Khoudh, Muscat, Sultanate of Oman

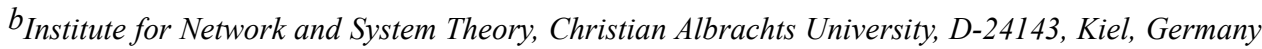

Received 6 April 2009; accepted 30 September 2009

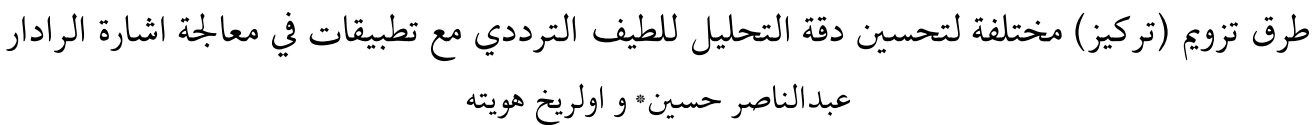

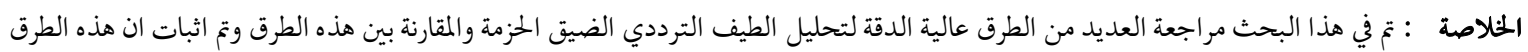

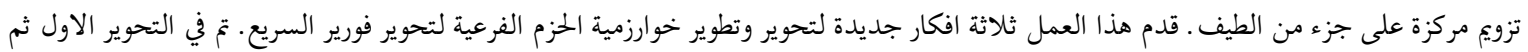

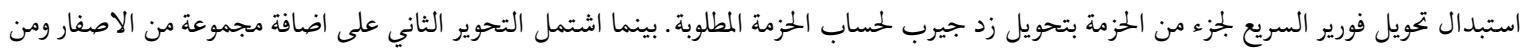

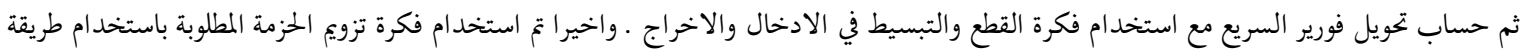

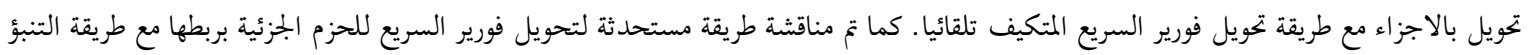

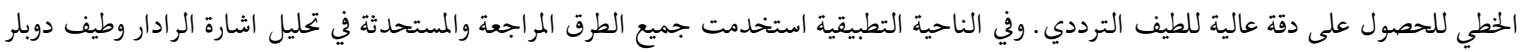

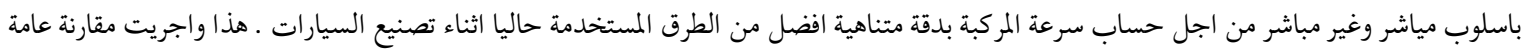

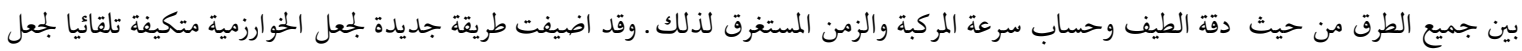
الحسابات النتعاقبة لسرعة المركبة اثناء السير مستقرة مع الخافظة على بساطة الخوارزمية. المفردات المفتاحية : طرق تحليل الطيف الترددي ، دقة الطيف المتناهي ، معالجة اشارة الرادار، حساب سرعة المركبات اثناء التصنيع ، خوارزميات متكيفة

\begin{abstract}
Different approaches for a high-resolution analysis of narrow-band spectra are reviewed and compared. Partial-band algorithms are proved to be zoom-FFT's. In this contribution, three new modifications of the (Subband-FFT) SB-FFT are presented. In the first modification the chirp z-transform substitutes the small FFT which calculates the band of interest. In the second modification, the idea of zero-padding the input signal is applied to the SB-FFT with pruning at both input and output. Lastly zooming a small band of frequencies using a method of transforming by parts is applied for a narrow-band signal using the adaptive SB-FFT. A newly introduced version of the subband technique is included also in this work. In this version the subband decomposition technique is combined with the linear prediction method for higher spectrum resolution. Application of the SB-FFT and its modified versions and the new version in measuring the Doppler-frequency directly and indirectly for the purpose of vehicle-speed measurements is introduced in this paper. Comparison between all methods in terms of complexity and resolution is given. A new idea of channel test is included to keep the real-time successive measurements of Doppler frequency stable and consistent as well as simple.
\end{abstract}

Keywords: Spectral analysis methods, High-resolution spectrum, Radar signal processing, Vehicle-speed measurements, Adaptive algorithms

\section{Introduction}

The discrete Fourier Transform (DFT) of a length-N sequence $\mathrm{x}(\mathrm{n})$ can be computed from

$$
X(k)=\sum_{n=0}^{N-1} x(n) W_{N}^{n k}
$$

The FFT is an efficient method for computing the FFT.

*Corresponding author's e-mail: abhossen@squ.edu.om
The spectrum of a real-valued signal obtained from applying; the FFT has a frequency range from 0 to $f_{s}$, / 2, with a frequency resolution $\Delta f=f_{s} / N$. While ss is the sampling frequency and must satisfy the condition $f_{s} / 2>f h$ (with fh denoting the highest frequency in the signal), the frequency resolution $\Delta . f$ can be written as (Mitra 2006 and Weeks 2007):

$$
\Delta f=\frac{2 \times f_{h}}{N}
$$


Higher resolution ( smaller $\Delta f$ ) can be obtained in one of two ways:

- Decreasing $f_{h}$, the highest frequency in the signal.

- Increasing $\mathrm{N}$, the transform length.

Zooming the FFT spectrum is a method to increase the resolution without decreasing the frequency limit or increasing the transform size. However, only a small part of the original range can be analyzed at a time (Markel, 1971; Sorensen et al. 1988; Rabiner et al. 1969; Shentov et al. 1991; Thrane, 1980; Hossen and Heute, 1993; Hossen and Heute, 2004; Harris 2004 and Keo and WSeng Gan, 2005). The two zoom-methods which are already used in spectrum analyzers, need to use a longer time signal in order to obtain higher-resolutions using Npoint FFT. These two methods are (Thrane 1980):

1. Zoom-FFT method 1 by frequency shift and low-pass filtration: This method is implemented normally in most FFT analyzers. The higher resolution is obtained by shifting (heterodyning) the frequency span of interest to fall around zero frequency, followed by a lowpass filter and down-sampling operation. The inputdata length must be multiplied by $Z$, if we want to improve the resolution by a zoom-factor $Z$. Large zoom-factors can be obtained using this method. Another similar approach is given by (Liu and Mintzer, 1978), in which no frequency shift is required and band-pass filters are used instead of low-pass filters.

2. Zoom-FFT method 2 by recording of a long time signal and transforming it by parts using a smaller transform: The higher resolution is obtained here by recording the necessary time signal $(Z \times N$ input samples) and performing $Z N$-point FFTs on successive parts of the input signal. This stage is followed by a phase compensation stage before adding the partial-frequency spectra to compensate for the time shift between the individual records. Another look at this stage shows that this method is nothing else but a radix- $Z$ FFT stage with pruning at the output to calculate only $N$ output samples. Another interpretation of this method is given as a partial-band FFT called transformdecomposition method with a modification using a technique similar to Goertzel's algorithm (Sorensen et al. 1988 and Sorensen and Burrus 1993).

The paper is organized as follows: The next section is a review material on SB-FFT. In section 3, different partial-band algorithms are discussed. Section 4 deals with different modifications of SB-FFT for the purpose of resolution improvements. Section 5 introduces the newly investigated version of SB-FFT combined with linear prediction technique. Section 6 shows applications of different approaches in radar signal processing for estimation of the Doppler spectrum for vehicle-speed measurements. Concluding remarks are given in section 7 .

\section{The SB-FFT}

The SB-FFT, which is an approximate FFT used with narrow-band signals, is one among other partial- band DFT algorithms such as the chirp z-transform method and the method of pruning and the transform decomposition method, which can find a narrow part of a full spectrum with high-resolution (same as the resolution obtained using a full length FFT). All these methods can be interpreted as a zoom-FFT similar to the zoom-FFT method 1 to find the band of interest with a high resolution.

In Fig. $1 a(n)$ and $b(n)$ are the low-pass and high-pass filtered versions of $x(n)$ as it is clear from their z-transforms $A(z)$ and $B(z)$ :

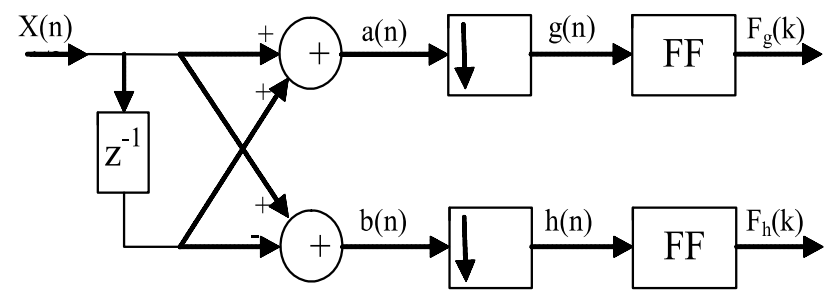

Figure 1. Two-band subband decomposition

$$
\begin{aligned}
& A(z)=\frac{1}{2}\left(1+z^{-1}\right) X(z) \\
& B(z)=\frac{1}{2}\left(1-z^{-1}\right) X(z)
\end{aligned}
$$

with $g(n)$ and $h(n)$ denoting their factor-2 down supplied versions, respectively:

$$
\begin{aligned}
& g(n)=\frac{1}{2}[x(2 n)+x(2 n+1)] \\
& h(n)=\frac{1}{2}[x(2 n)-x(2 n+1)]
\end{aligned}
$$

The full-band size- $N$ DFT $X(k)$ can be obtained by (Shentov et al. 1991 and Hossen and Heute, 2004):

$$
x(k)=\left(1+W_{N}^{k}\right) F_{g}(k)+\left(1-W_{N}^{k}\right) F_{h}(k)
$$

Eq. (5) is approximated for calculating only the lowpass band corresponding to the upper-part of Fig. 1:

$$
x(k) \approx\left(1+W_{N}^{k}\right) F_{g}(k), k \in(0,1, . ., N / 4-1)
$$

The decomposition process in Fig. 1 can be repeated $\mathrm{m}$ times to get $M=2^{\mathrm{m}}$ subbands, out of which only one band is to be computed depending on information known or derived adaptively about the input signal power distribution (Hossen and Heute, 1993). In other words, the filtering decomposition of Eq. (4) can be repeated to $g(n)$ and $h(n)$ and the approximation done for the half-band case can be applied to calculate one out of M-bands. If we have no information about the input signal, a method of bandselection which was introduced in (Hossen and Heute, 
1993) can be used. This method corresponds to finding $\operatorname{sgn}(A)$ for a 2-band decomposition:

$$
\operatorname{sgn}(A)=\operatorname{sgn} \sum_{n=0}^{\frac{N}{2}-1}|g(n)|-|h(n)|,
$$

and according to the sign of $A$, we decide either to follow the low-frequency part if $\mathrm{A}$ is positive, or the high-requency part if $A$ is negative, while the other part is ignored.

\section{Resolution Improvements Using Partial- Band Algorithms}

Four different methods are investigated here. In all these methods the number of input time samples is fixed to $N$. Complexity of those algorithms is explained in terms of the number of complex multiplications.

\subsection{The Chirp Z-Transform}

The chirp z-transform enables the evaluation of the ztransform of $N$ time samples at $P$ equiangularly spaced points on contours which spiral in or out (circles being a special case) from an arbitrary starting point in the z-plane (Liu and Mintzer, 1978). The flexibility in the chirp ztransform is that $N$ needs not to be the same as $P$, and the output-point spacing is arbitrary. The chirp z-transform is defined as:

$$
X\left(z_{k}\right)=\sum_{n=0}^{N-1} x(n) A^{-n} w^{n k}, \quad k=0,1, \ldots, p-1
$$

while $z^{k}=A w^{-k}$ This provides an efficient algorithm for calculating $X\left(z^{k}\right)$ by implementing it as a discrete convolution. In Eq. (8) $A=A_{0} \exp \left(-j 2 \pi \theta_{0}\right)$ and $w=w_{0} \exp$ $\left(-j 2 \pi \phi_{0}\right)$ are both complex constants. If $w_{0}=1$, the spiral is a circular arc, and if $A_{0}=1$ this circular arc is the unit circle, and if $\theta_{0}=1$ the first point is the $(1,0)$ point. Choosing the z-plane contour on the unit circle and selecting an initial point of the band of interest, the spacing between output points can be defined arbitrarily.

If the total number of input points is $N$, and the total number of calculated points is $P$, then the number of complex multiplications will be $Q\left(\frac{3}{2} l d Q+4\right)$, where $Q=N+P-1$.

\subsection{FFT-Pruning}

Pruning the decimation in time or decimation in frequency (Mitra, 2006) FFT corresponds to eliminating operations that do not contribute to the output. In (Juo and W-Seng Gan, 2005) a pruning method is proposed which involves frequency shifts to calculate any number of adjacent transform outputs. A method for partial spectrum computation introduced by Wild (Lie and Mintzer 1978) is similar to that used in (Sorensen and Burrus 1993). The number of complex multiplications in case of DIT output pruning are $\frac{N}{2} l d P+(N-P)$, where $1 d p$ is $\log _{2} p$. Pruning can also be performed with the split-radix FFT (Roche, 1992).

\subsection{Transform-Decomposition}

This partial-band algorithm has the same idea as the zoom-FFT method 2. This idea is modified (Sorensen et al. 1988 and Sorensen and Burrus, 1993) to be based on a mixture of a standard FFT algorithm and a computational structure similar to that, used in Goertzel's algorithm. The FFT's can he performed in this method using the three-butterfly split-radix FFT (Sorensen et al. 1986), which requires the lowest number of operations of any known power of two FFT. The number of complex multiplications in such a split-radix FFT is known to be $\frac{N}{3} l d N-N+\frac{4}{3}$. The total number of complex multiplication in the transform decomposition method is $\frac{N}{P}\left(\frac{P}{3} l d P-P+\frac{4}{3}\right)+(N-P)$.

\subsection{SB-FFT}

Only the band of interest is calculated using a small-length FFT after decomposing the input signal in many possible bands. This method has the advantage that using the adaptive algorithm in (Hossen and Heute, 1993), briefly stated in the previous section, we can select the band to be calculated in parallel to the decomposition stages of the algorithm. The difference between this approximate method and the first three methods is that the SB-FFT can be used only for a narrow-band signal, but in this case is extremely efficient. The number of complex multiplications in this method is $\frac{N}{2 M} l d N$ when a Radix-2/DIT algorithm is applied. This figure may be further reduced if the threebutterfly split-radix FFT is used.

The same resolution is obtained in all four methods, if the total input number and the calculated output number of points are the same. Figure 2 shows the application of all four methods with $\mathrm{N}=1024$ in detecting two sinusoids (of 35 and $37 \mathrm{~Hz}$ ) in wide-hand noise with $\mathrm{SNR}=-3 \mathrm{~dB}$; the sampling rate in this case was $f_{S}=1024 \mathrm{~Hz}$. The resolution is found to be $\Delta f=1 \mathrm{~Hz}$. The first $N_{c}=128$ points are calculated. The $S N R$ that still allows for detecting the two sinusoids, is found to be around $S N R=-15 \mathrm{~dB}$.

\section{Modifications of SB-FFT for Purpose of Resolution Improvements}

\subsection{SB-Filter with Chirp z-Transform}

A combination between the first and the fourth methods yields a new method, in which the resolution improvement is increased: The small FFT in Fig. 1 can be substituted with a chirp z-transform. The first point can be 

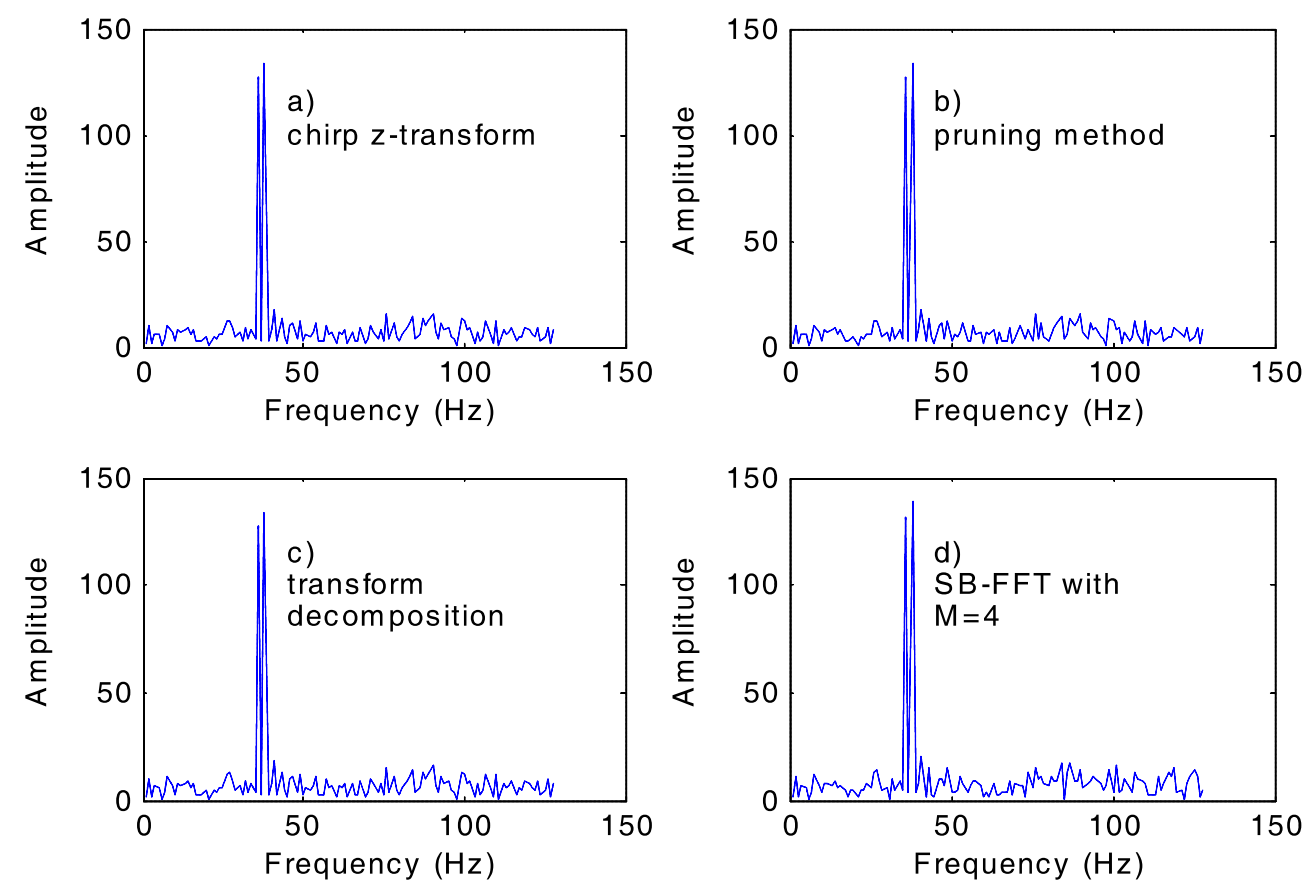

Figure 2. Sinusoids detection with different partial-band algorithms

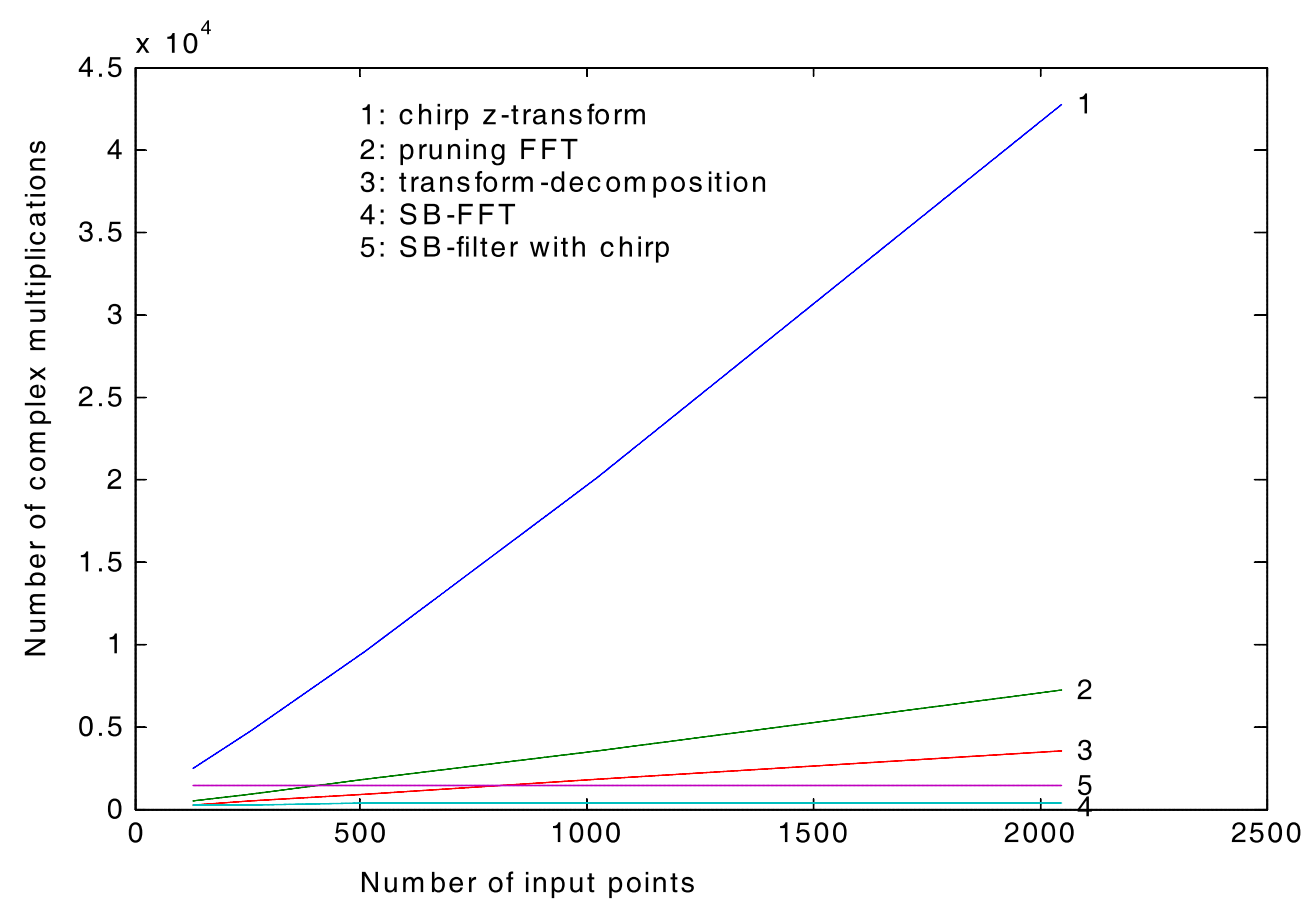

Figure 3. Complexity comparison between diffeerent partial band approaches

selected with the adaptive method inherent in the SB-FFT of (Hossen and Heute, 1993), the spacing between the points in the selected band can be decreased to obtain a higher resolution. The number of complex multiplications is $V\left(\frac{3}{2} l d V+4\right)$ where $V=\frac{N}{M}+P-1$, where $P$ is the number of points to be calculated.

A complexity comparison between this method and the other four methods described in section 3 is shown in Fig.
3 in terms of the number of complex multiplications required to compute $P=32$ point when the total input points vary from 128 to 1024 . Fig. 4.a shows the application of this methods with $N=1024$ in detecting two sinusoids (of 15 and $17 \mathrm{~Hz}$ ) in wide-hand noise, the sampling rate in this case was $f_{S}=1024 \mathrm{~Hz}$. The resolution is found to be $\Delta f=1 H z$. The first $N_{c}=128$ points are calculated. For this example the required number of complex multiplication is 4963 . This method can be improved regarding resolution, if the spacing between the calculated points is 
changed from $\frac{2 \pi}{N}$ to $\frac{\pi}{N} . \Delta f$ is found to be $0.5 \mathrm{~Hz}$. Fig. $4 \mathrm{~b}$ shows the same example of Fig. $4 \mathrm{a}$ after this improvement to detect two sinusoids (of 15 and 16 $\mathrm{Hz}$ ). No additional complex multiplication is required. Using the adaptive capability in SB-FFT needs no extra complex multiplications. Of course it requires some extra time, but it is shown in (Hossen and Heute, 1993) that the adaptive SB-FFT is still faster than other partial-band algorithms.

\subsection{SB-FFT with Zero-Padding}

Zero-padding the input-signal (though only in the sense of a spectral interpolation) and then applying any partialband algorithm to find the transform of a selected band can improve the resolution.

For the corresponding longer DFT, however, not all the output points need to be calculated, only $P=2 l f$ points between, say, $F 1$ and $F 2$. A radix-2 FFT is used with both pruning at the input because of the zeros and pruning at the output because only a limited band is to be calculated (Sreenivas 1980).

A new modification to the SB-FFT algorithm is found to be possible, in which we pad the input signal with a number of zeros and then apply the SB-FFT decomposition. Pruning can be applied in the Hadamard-part and at the input of the small FFT because of the zeros and at the output of the small FFT because not all the outputs are required. The number of complex multiplications is given by:

$$
M u l=\frac{N}{2 M}(1+l f-m+1)+\left(\frac{N}{M}-P\right)+\frac{N}{2 M}
$$

Figure 4c shows the result of zero-padding 1024 input points to 2048 points and then calculating only the first 1024 point using pruning at the input and at the output of the FFT algorithm. The resolution is found to be $\Delta f=$ $0.5 \mathrm{~Hz}$. The same two sinusoids in Fig. $3 \mathrm{~b}$ are used.

\subsection{SB-Zoom FFT}

1. SB-FFT interpretation as zoom-FFT method 1:

2. Considering this approach, Fig.1 shows that the twoband SB-FFT is a zoom-FFT (with factor 2) following the idea of zoom-FFT method 1 for the low-frequency part Using the adaptive SB-FFT we can zoom any band of interest, the zoom-factor equaling the number of the decomposition bands. The low-pass filters inherent in the zoom-method are replaced by the simple first order low-pass or high-pass filters of the SBFFT method. The choice of low-pass or high-pass is done in each step with the help of the adaptive algorithm. A modification of the SB-FFT is done here such that in each stage of the decomposition and according to the result of Eq. (7) we perform phase compensation, if a high pass reduction stage must be followed, and then a low-pass filter is used only. The phase compensation done at each high-pass stage makes the SB-zoom processor more complex.

3. Combination of SB-FFT and Zoom FFT method 2:
A combination between the SB-FFT and Zoom method 2 yields a method used to analyze a part of the band of interest in a narrow-band signal. This method can be applied as follows:

- $\quad$ Recording a long-time signal;

- $\quad$ SB-filtering (applying Eq. (4));

- Transforming the resulting signal by parts.

The SB-filters can also use the adaptive capability to select the band to be transformed by parts. The result of applying 4096 points with Zoom factor $=4$ and SB-FFT with 4 bands to detect the same two sinusoids in Fig.4a is shown in Fig. $4 \mathrm{~d}$. The resolution in this example is $\Delta f=$ $0.25 \mathrm{~Hz}$. The spectrum shows only the first quarter of the low-low band.

\section{SB-Zoom FFT with Linear Prediction}

Parametric spectral estimation is a three-step procedure. The first step is to select a model. The second step is to estimate the parameters of the assumed model using the available data samples. The third step is to obtain the spectral estimate by substituting the estimated model parameters into the theoretical PSD implied by the model (Key and Steven, 1988).

A parametric technique is used to find the parameters of a mathematical model describing the time signal for high-resolution spectral estimation. Linear prediction yields an Auto-Regressive (AR) process which models a signal as the impulse response of all-pole filter. It assumes that each output sample $x(n)$ is a linear combination of the past $n$ outputs, and that the coefficients are constant from sample to sample:

$$
x(n)=-\sum_{k=1}^{p} a(k) x(n-k),
$$

where $x(n)$ is the signal sequence to which the AR-model of Pth order is to be found by, eg. Forward Linear Prediction (FLP) depending on the past samples. The difference between the signal values and the estimated values is considered as an error of the FLP process:

$$
e_{f p}(n)=x(n)+\sum_{k=1}^{p} a_{f p}(k) x(n-k)
$$

If the output sample of the estimation process is found using a linear combination of the next $p$ outputs, the process is called Backward Linear Prediction (BLP) and results in the following error:

$$
e_{b p}(n)=x(n-p)+\sum_{k=1}^{p} a_{b p}(k) x(n-p+k)
$$

If both forward and backward linear predictions are combined to find the parameters of the model, the process is called Forward-Backward Linear Prediction (FELP), and the sum of squares of the errors given by Eqs. $(11,12)$ is tested for the minimum value to find the coefficients of the model filter: 

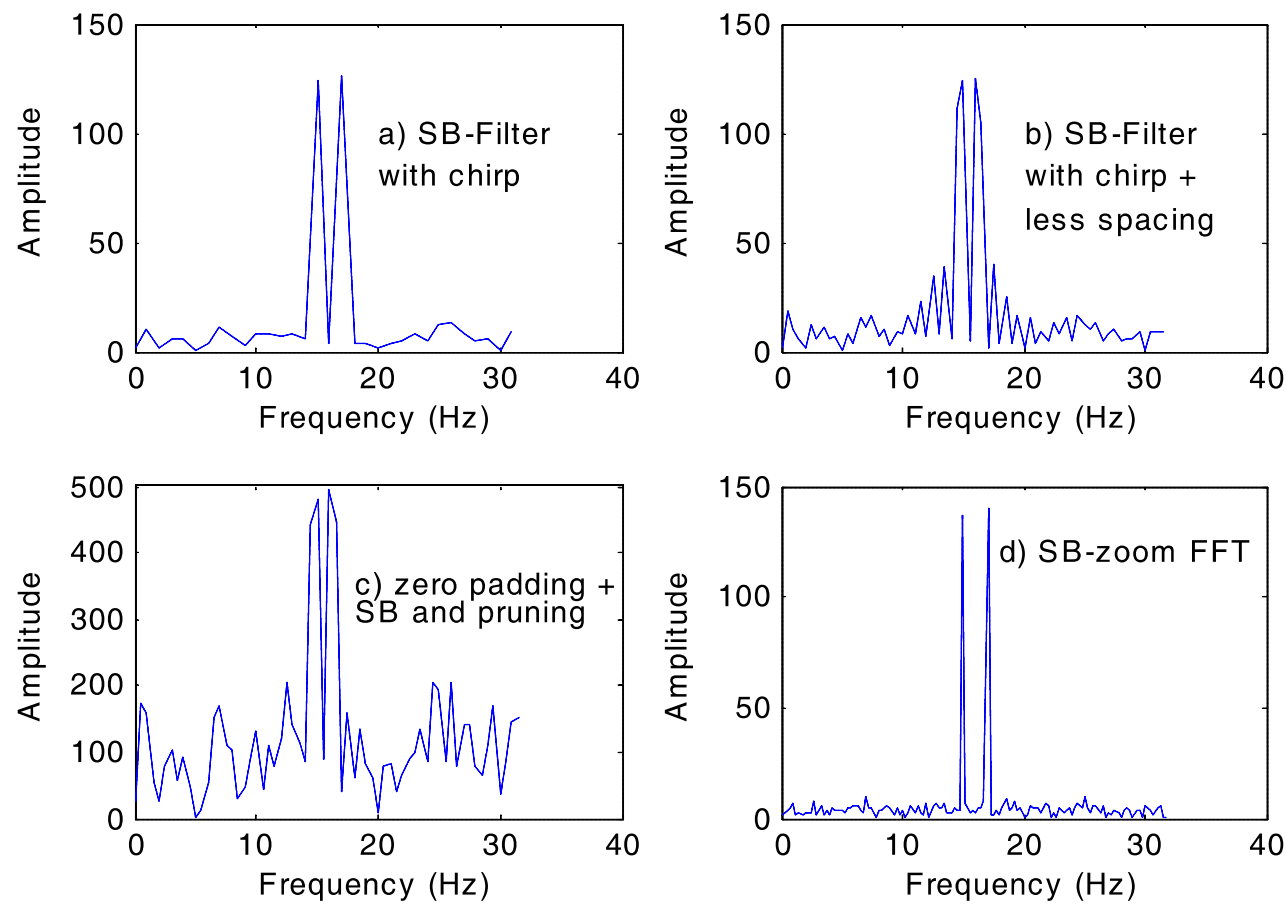

Figure 4. Different modifications of SB-FFT for resolution improvements
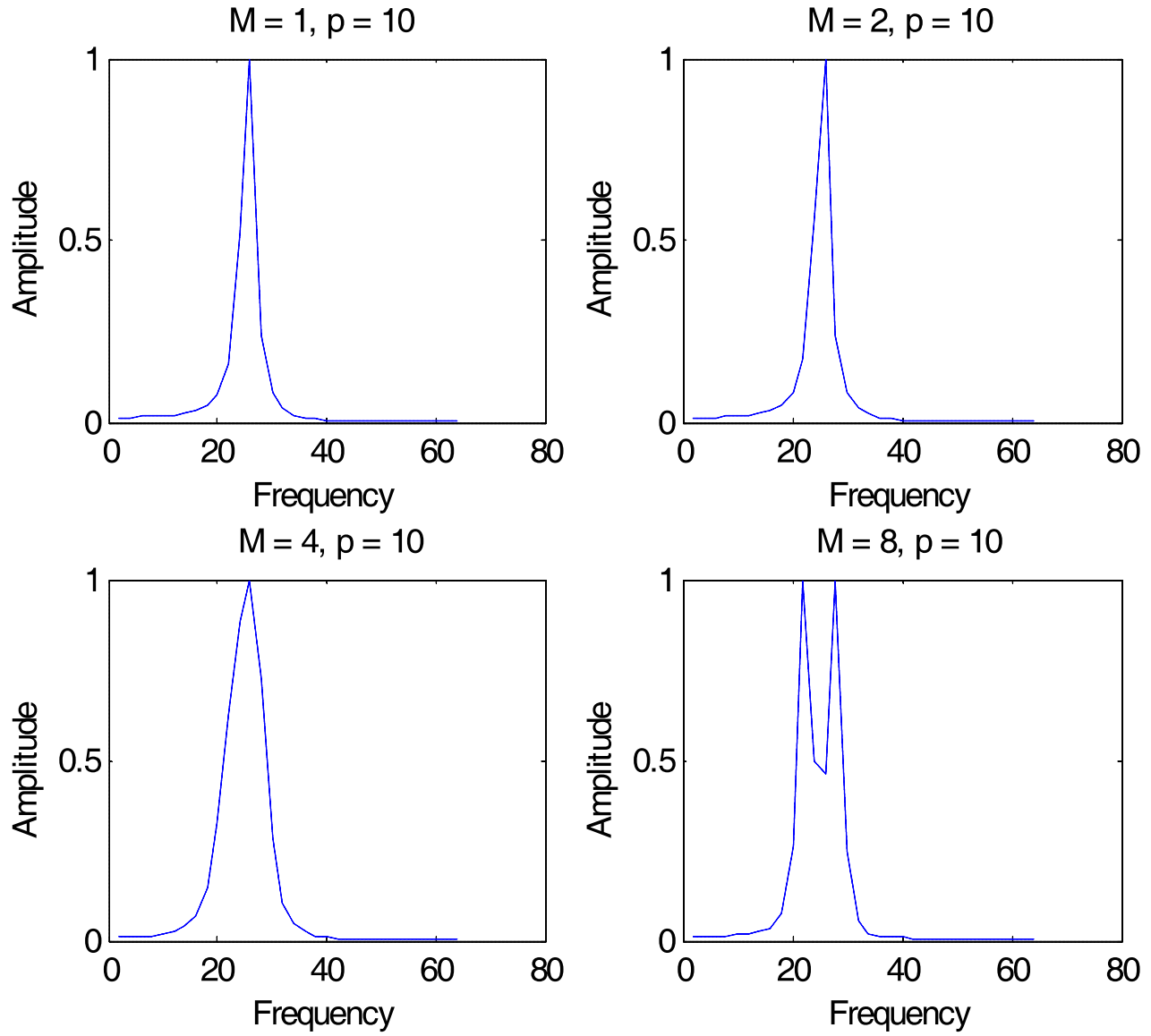

Figure 5. SB-FFT with different number of subbands combined with linear prediction 


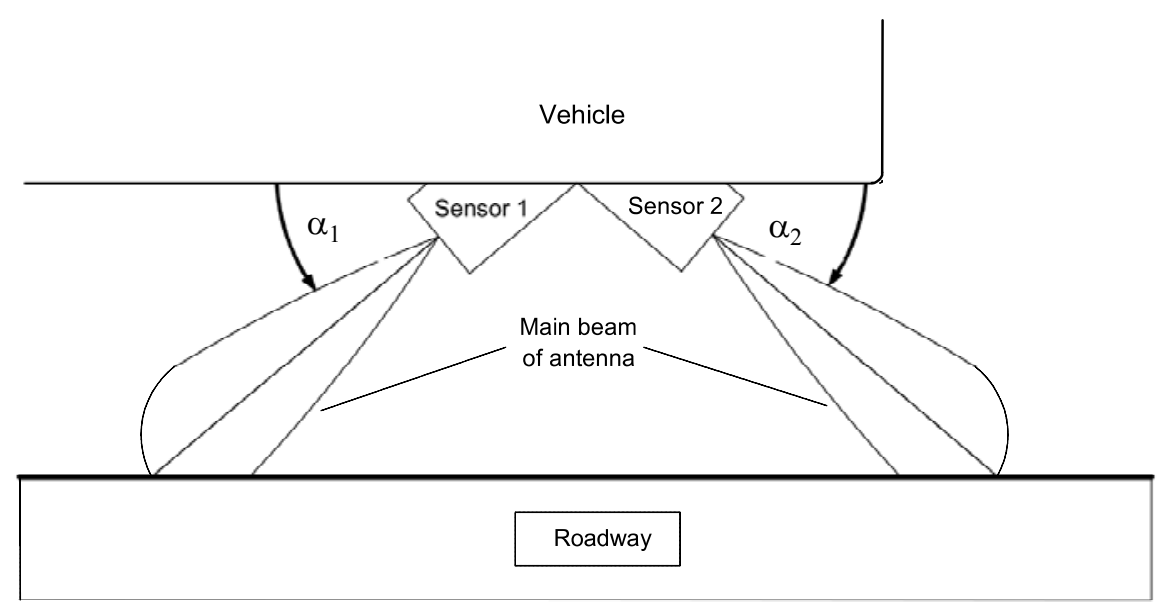

Figure 6. Janus configuration of the sensors for doppler radar experiment

$$
\sum_{n}\left(e_{f p}(n)\right)^{2}+\sum_{n}\left(e_{b p}(n)\right)^{2}=\operatorname{Min}
$$

In linear prediction, the autocorrelation method of autoregressive (AR) modeling can be used to find the filter coefficients. This technique is called the maximum entropy method (MEM) of spectral estimation (Marples and Lawrence, 1987; Matlab Signal Processing Toolbox, 1996 and Kleinhempel et al. 1992). After the coefficients of the digital filter are found, the frequency response of the filter (equivalent to the frequency spectrum of the time series $x$ ) can also be found by classical spectrum-analysis algorithms. The new zoom technique is obtained by (Sreenivas and Rao, 1980):

1. Subband decomposition of the input signal applying the simple filters in Fig. 1. The decomposition can be repeated till finding the band of interest.

2. Calculating the a coefficients of the IIR filter from Eq. (10) from the result of the previous step by selecting an order $\mathrm{p}$.

3. Calculating the frequency spectrum using the resulted a coefficients.

In Fig. 5, the spectrum of two adjacent frequencies 20 $\mathrm{Hz}$ and $25 \mathrm{~Hz}$ is found for a sampling frequency of 1000 $\mathrm{Hz}$ and a linear prediction of order $\mathrm{p}=10$ by the following different methods:

1. Fullband case $M=1$, direct linear prediction for a total number of $N=256$ points.

2. Subband decomposition $M=2$, followed by linear prediction.

3. Subband decomposition $M=4$, followed by linear prediction.

4. Subband decomposition $M=8$, followed by linear prediction.

\section{Results of Radar Signal Processing Appli- cations}

A Doppler-radar sensor for measurements of the socalled "True Speed Over Ground" (TSOG), based on mil- limeter-wave frontends $(61 \mathrm{GHz})$ and digital processing of baseband signals, was presented in (Kleinhempel et al. 1992; Claeben 1991 and Besson and Castanie 1990). . The task of the digital processing is to determine the Doppler frequency, which is proportional to TSOG. Two millimeter-wave sensors are mounted at the bottom side of the vehicle in a Janus configuration, see Fig. 6. After reflection on the roadway the signal reaching the sensor is shifted by the Doppler frequency $f_{d}$;

$$
f_{d i}=\frac{2 v f_{o} \cos \left(a_{i}+(-1)^{i} \theta\right.}{c}
$$

with

i : sensor number,

$v$ : velocity of vehicle,

$\alpha_{i}$ : angle of inclination of each sensor,

$\theta$ : actual pitch angle, ie. angle between the road and the bottom of the car,

$c$ : light-velocity,

$f_{O}:$ transmitter-frequency.

To determine $v$, the Doppler frequencies $f_{d 1}$ and $f_{d 2}$ should be firstly estimated and then the pitch angle can be calculated;

$$
\theta=\tan ^{-1}\left(\frac{f_{d 1} \cos \alpha_{2}-f_{d 2} \cos \alpha_{1}}{f_{d 1} \sin \alpha_{2}+f_{d 2} \sin \alpha_{1}}\right)
$$

Now $v$ can be found from:

$$
v=\frac{0.5 c\left(f_{d 1}+f_{d 2}\right)}{f_{0}\left[\cos \theta\left(\cos \alpha_{1}+\cos \alpha_{2}\right)+\sin \theta\left(\sin \alpha_{1}-\sin \alpha_{2}\right)\right]}
$$

The estimation of the Doppler frequencies fdi can be simply done by using a Fast Fourier Transform (FFT) algorithm and finding the position of the maximum of the Doppler spectrum (Richards, 2005; Mahafza 2008; Mahafza and Elsherbeni 2003 and Blanchet and Charbit, 2006). The spectral analysis based on Fourier-transform has two disadvantages: bad resolution with short signal lengths and large variance of the estimated spectrum. 
The rugged structure of the spectral maximum of the Doppler radar depends on the condition of the roadway. So applying the FFT directly results sometimes in nonaccurate estimation of the Doppler-frequency. A zoom. FFT can help in gaining higher spectral resolution, since the spectrum is evaluated in a narrow frequency band of interest. Parametric techniques can be also used for higher spectral resolution. Different methods for simplifying the digital signal processing part of the vehicle-speed measuring system based on Doppler radar are to be discussed in this section. Some of these methods are direct (only transform methods based on SB-FFT or its modification) or indirect (combined parametric spectral analysis with the subband technique).

\subsection{Direct Methods}

An FFT can be applied directly to the sections of the input signal, and the Doppler frequency can be determined at each time from the spectral maximum. The process is repeated every $40 . .80 \mathrm{~ms}$, and from the two frequencies measured at both sensors, the velocity can be estimated using Eq. (16). This may be compared with a highly accurate reference velocity, measured with a so-called peiseler whee (Claeben 1991). The difference between the estimated velocity and the reference is considered as an error. The accuracies of applying the following direct methods (in terms of the mean error me and the standard deviation $s d)$ to a Doppler signal results from a car driving on a dry roadway with a speed of $20 \mathrm{~km} / \mathrm{h}$ are given in Table 1:

1. CT-FFT (2048 points),

2. adaptive SB-FFT,

3. adaptive SB-filter + Chirp transform with spacing $\pi$ / N.

The same resolution is obtained in all partial-band algorithms described in section 3 , if the total input number and the calculated output number of points are the same. This means that if we apply any one of the partial band algorithms described in section 3 will result in the same accuracy of using the SB-FFT, which is also similar to apply full-band FFT as seen in Table 1.

Table 1. Accuracy of direct methods in $\mathrm{km} / \mathrm{h}$

\begin{tabular}{|c|c|c|}
\hline Method & me & sd \\
\hline FFT & -0.174 & 0.140 \\
\hline SB-FFT & -0.17 & 0.140 \\
\hline SB-filter + chirp & -0.186 & 0.12 \\
\hline
\end{tabular}

6.2 Indirect Methods using Parametric Techniques

The Doppler signal from on-board radar, used to estimate the speed of a train (Besson, $\mathrm{O}$ and Castanie, F, 1990) may be simply represented by a sine-wave amplitude modulated by a random lowpass process:

$$
x(n)=y(n) \cos \left(2 \pi f_{d} n+\psi\right)
$$

where $\psi$ is a random variable uniformly distributed from [0 to $2 \pi$ ] and $y(n)$ can be modeled by an (AR) process.
The parametric techniques for spectral estimation consist of the following steps;

1. Choosing a suitable time model to approximate the data under consideration

2. Estimating the parameters of the model (coefficients of the prediction error filter)

3. Finding the transfer function of the prediction error filter

4. Finding the frequencies of the sine components from the positions of the zeros of the transfer function found above.

Then the Doppler frequency $f_{d}$ can be found from the mean value of the estimated AR- frequencies. The expected Doppler frequencies are between 0 and about $20 \mathrm{kHz}$, corresponding to vehicle speeds up to $250 \mathrm{~km} / \mathrm{h}$ and inclination angles $\alpha_{i}$ of approximately 45 degree. More accurate estimations can be done with the combination of a pre-processor zoom and conventional AR-model spectral estimators (Hossen and Heute, 1994). In (Hossen and Heute, 1994) the determination of $f d$ is done using, eg. the FBLP technique. Figure 7 shows such a block for the pre-processor zoom for estimating $f_{d}$ from the radar timesignal. Three different model orders $p$ are used. For the initialization process, $p=20$ is applied, for the demodulated signal's path $p=2$, for the undemodulated-signal's path $p=3$. The decision whether demodulation is required or not, is obtained by comparing fd with a reference value $f_{r}$.

The results obtained for this pre-processor zoom method and its following modifications using the subband approach are given in Table 2, for the same example of a car driving on a dry roadway with a speed of $20 \mathrm{~km} / \mathrm{h}$.

Table 2. Accuracy of indirect methods in $\mathrm{km} / \mathrm{h}$

\begin{tabular}{|c|c|c|}
\hline Method & me & Sd \\
\hline Pre-Processor Zoom & -0.1173 & 0.0544 \\
\hline Modified method 1 & -0.1171 & 0,0541 \\
\hline Modified method 2, M $=2$ & -0.188 & 0.063 \\
\hline Modified method 2, M =4 & $-0,207$ & 0.063 \\
\hline Modified method 3, M =2 & $-0,096$ & 0.059 \\
\hline Modified method 3, M = 4 & -0.032 & 0,073 \\
\hline
\end{tabular}

1. First modification: Only the initialization process is replaced by an adaptive SB-FFT with $M=8$, and then $\mathrm{fd}$ is found from the spectral maximum.

2. Second modification: The pre-processor is replaced by SB-filters, since the SB-FFT can be interpreted as a zoom-FFT (Claeben 1991). Then, to determine $f_{d}$, the AR-model is used with $p=2$ always. Both halfband $(M=2)$ and quarter-band $(M=4)$ SB-filters are investigated,

3. Third modification: After the pre-processor zoom, we perform SB-filters with $M=2$ or $M=4$, and then the AR-model is applied to find the FFT with fewer points. 


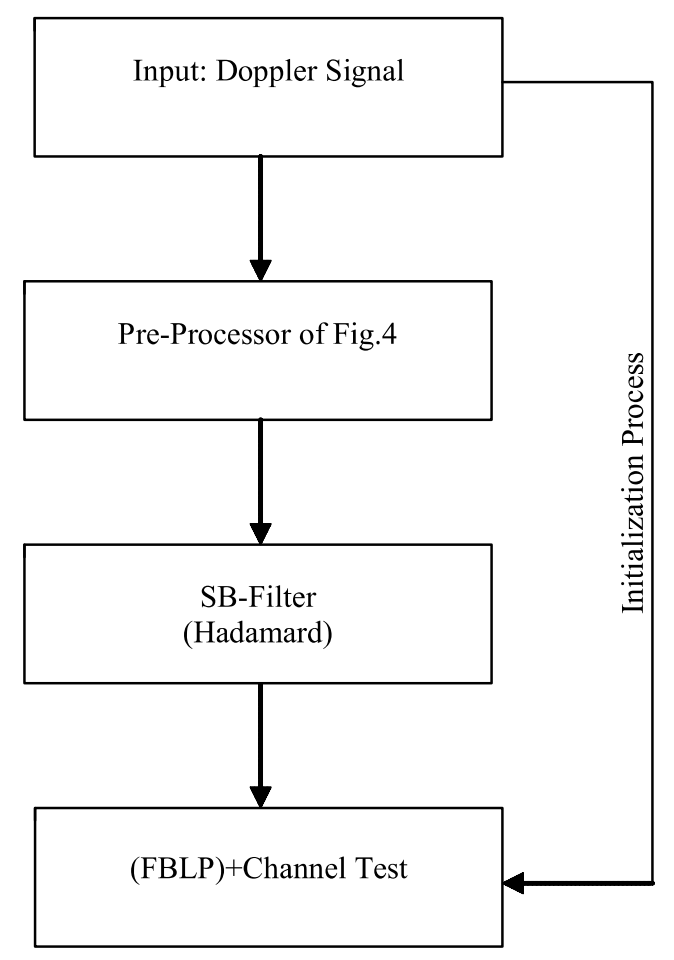

Figure 7. Doppler-frequency estimation using a pre-processor zoom

The results in Table 2 for the three different modifications are good approximations to the results of the method explained of a pre-processor zoom though with some larger errors. Since the width of the spectral peak depends on the speed of the car, for higher speed it will be broader. Also if the roadway is wet, then the amplitude of the reflected signal is low and the signal-to-noise ratio is also low. The "channel test" of the following section is able to decrease this sensitivity.

\subsection{Indirect Method with Channel Test}

Using the SB-filters in the third modification of the last subsection with a higher value of $M$, results in a fast estimation of the Doppler frequency but with less accuracy. If we keep using this higher value of $M$ to measure successive values of the Doppler frequency, we may end with unacceptable values and unstable results. In order to keep better accuracy and to have a sort of adaptive capability of keeping our measurement system stable, an idea of channel test is implemented in this real-time application, in which successive measurements are needed. In order to test whether the calculated Doppler frequency at any measuring time $k$ $\left(f_{d}(k)\right)$ is correct, a weighted-mean value $\hat{f}_{d}(k)$ is calculated from the last three calculated values as below:

$$
\hat{f}_{d}(k)=\left[3 f_{d}(k-1)+2 f_{d}(k-2)+f_{d}(k-3)\right] / 6
$$

The absolute value of the difference between $f_{d}(k)$ and $\hat{f}_{d}(k)$ is found and compared with a specified

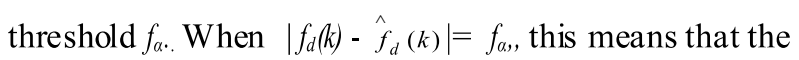
measured frequency value is acceptable, and we perform the next measuring with the same approximation (using the same value of $M$ in the subband filter), but when $\left|f_{d}(k)-\hat{f}_{d}(k)\right|>f_{a}$, then the measured value is incorrect and to be corrected to the previous value $f_{d}(k-1)$, and in the next measuring, a smaller value of $M$ is selected. The channel test is included with the modified method of 3 of the previous section with number of subbands $M=4$ and $M=8$ changed to 2 , whenever the result of the channel test shows an incorrect result. This method is used with three different driving situations (dry roadway with speeds of $20 \mathrm{~km} / \mathrm{h}, 30 \mathrm{~km} / \mathrm{h}$, and $70 \mathrm{~km} / \mathrm{h})$. The results of this improved method are listed in Table 3. The channel test idea is illustrated in Fig. 8.

\section{Concluding Remarks}

Several different approaches for improving the spectral resolution are investigated. All partial-band algorithms are interpreted as zoom-FFTs. The approximated SB-FFT is shown to be of less complexity than all the other methods. A new approach is suggested, in which the SB-FFT is combined with the chirp z-transform. This new approach is shown to have a better resolution than other methods. The SB-FFT method is made more efficient by applying pruning both at the input and the output after zero-padding the input signal to obtain a higher resolution. Also the 


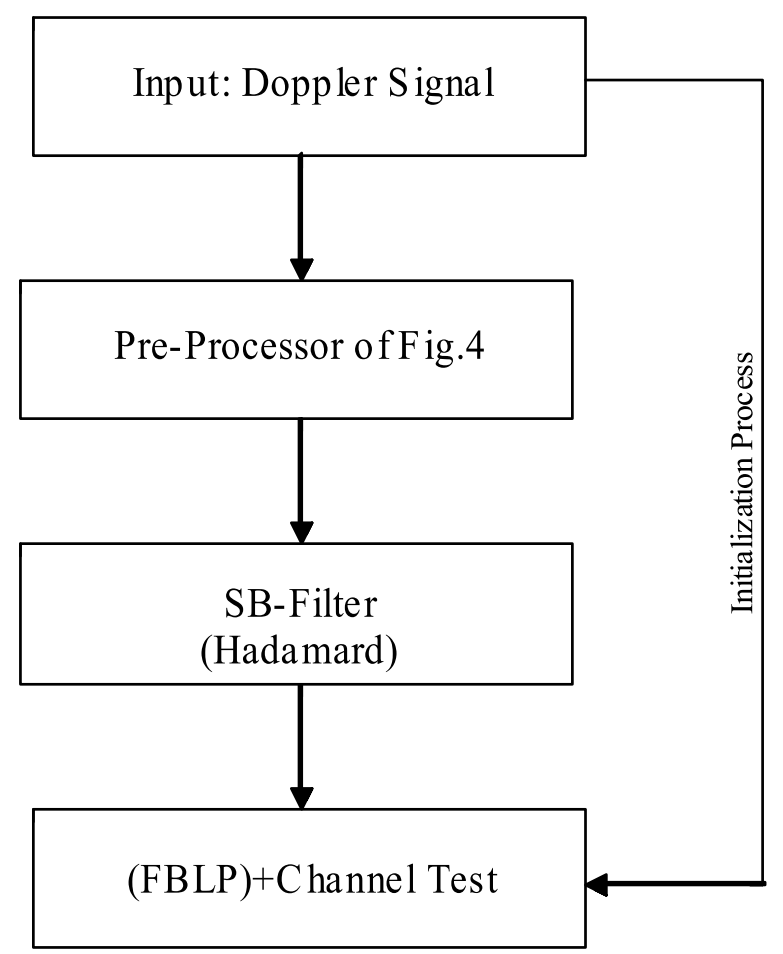

Figure 8. Doppler-frequency estimation using indirect method and channel test

transform decomposition method is applied with the SBFFT to find a part of the interesting band with a high resolution.

Table 3. Accuracy of indirect methods in $\mathrm{km} / \mathrm{h}$ with channel test

\begin{tabular}{|c|c|c|}
\hline Method & $\boldsymbol{m} \boldsymbol{e}$ & $\boldsymbol{S} \boldsymbol{d}$ \\
\hline $\mathrm{SB}(\mathrm{M}=4, \mathrm{M}=2), 20 \mathrm{~km} / \mathrm{h}$ & -0.0265 & 0.0728 \\
\hline $\mathrm{SB}(\mathrm{M}=8, \mathrm{M}=2), 20 \mathrm{~km} / \mathrm{h}$ & -0.0562 & 0,0938 \\
\hline $\mathrm{SB}(\mathrm{M}=4, \mathrm{M}=2), 30 \mathrm{~km} / \mathrm{h}$ & -0.119 & 0.1387 \\
\hline $\mathrm{SB}(\mathrm{M}=8, \mathrm{M}=2), 30 \mathrm{~km} / \mathrm{h}$ & -0.087 & 0.2245 \\
\hline $\mathrm{SB}(\mathrm{M}=4, \mathrm{M}=2), 70 \mathrm{~km} / \mathrm{h}$ & -0.050 & 0.295 \\
\hline $\mathrm{BB}(\mathrm{M}=8, \mathrm{M}=2), 70 \mathrm{~km} / \mathrm{h}$ & -0.1901 & 0.3033 \\
\hline
\end{tabular}

The evaluation of Doppler-radar signals in a real-time application for the purpose of vehicle-speed measurements is approximated in this work by using the SB-FFT instead of the CT-FFT. The SB-FFT is applied directly to find the speed of the vehicle by analyzing a Doppler spectrum of two millimeter-wave sensors mounted at the bottom side of the vehicle. The combination of both SB-filters and chirp z-transform is also used for better resolution. The subband approach is also combined with linear prediction methods to find the Doppler frequencies more accurately. A novel idea of channel test is added to insure that the accuracy of the measurement system is kept high while the complexity of the technique is reduced using SB techniques instead of full-band techniques.

\section{References}

Besson, O. and Castanie, F., 1990, "Doppler Frequency Estimator Performance Analysis," Proceedings ICASSP'90, Albuguerque.

Blanchet, G. and Charbit, M., 2006, "Digital Signal and Image Processing using MATLAB," Wiley-ISTE.

Claeben, S., 1991, "Untersuchung Zur Auswertung Von Dopplersignalen Mit Parametrichen Spektralanalyseverfahren," Diplomarheit, AG Digitale Signalverarbeitung, Ruhr- Universität Bochum (in German).

Harris, F.J., 2004, "Multirate Signal Processing," Prentice Hall.

Hossen, A.N. and Heute, U., 1993, "Fully Adaptive Evaluation of Sub-Band DFT," Proc. Of IEEE Int. Symp. on Circuits and Syst., Chicago, pp. 655-658.

Hossen, A.N. and Heute, U., 1994, "Different Approaches for a High-Resolution Narrow-Band Spectrum," Proceedings of EUSIPCO'94, Edinburgh, pp. 17161719.

Hossen, A. and Heute, U., 2004, "Parametric Modeling of Decomposed Sub-bands: Resolution Improvement and Applications for Narrow-Band Signals," Signal Processing Journal, Vol. 84, pp. 2195-2206.

Kay Steven, M., 1988, "Modern Spectral Estimation Theory and Applications," Prentice Hall.

Kleinhempel, W., Stammler, W. and Bergmann, D., 1992" Radar Signal Processing for Vehicle Speed Measurements," EUSIPCO-92, pp. 1829-1832.

Kuo, S.M. and W-Seng Gan, 2005, "Digital Signal Processing, Architecture, Implementations, and applications," Pearson Education. 
Liu, B. and F. Mintzer, 1978, "Calculation of NarrowBand Spectra by Direct Decimation," IEEE Trans. Acoust., Speech, Signal Processing, Vol. ASSP-26(6), pp. 529-534.

Mahafza, B.R. and Elsherbeni, A., 2003, "MATLAB Simulations of Radar System Design," Chapman \& Hall/CRC.

Mahafza, B.R., 2008, "Radar Signal Analysis and Processing using MATLAB," Chapman \& Hall/CRC.

Markel, J.D., 1971, "FFT Pruning," IEEE Trans. Audio and Electroacoustics," Vol. AU-19(4), pp. 305-311.

Marple Lawrence, S., 1987, "Digital Spectral Analysis with Applications," Prentice Hall.

"Matlab Signal Processing Toolbox," 1996, The MathWorks.

Mitra, S.K., 2006, "Digital Signal Processing, A Computer Based Approach," McGraw-Hill.

Nagai, K., 1986, "Pruning the Decimation-in-time FFT Algorithm with Frequency Shift," IEEE Trans. Acoust., Speech, Signal Processing, VoI. ASSP-34(4), pp. 1008-1010.

Rabiner, L. and R. Schafer., 1969, "The Chirp zTransform Algorithm and Its Application," Bell Systems, Tech. J. Vol. 48, pp. 1249-1292.

Rabiner, L.R., Schafer, R.W. and Rader, C.M., 1969, "The Chirp Z-Transform Algorithm and its Application," Bell Syst. Tech. J., Vo1. 48, pp. 12491292.

Richards, M.A., 2005, "Fundamentals of Radar Signal Processing," McGraw-Hill.

Roche, C., 1992, "A Split-Radix Partial Input/Output Fast
Fourier Transform Algorithm," IEEE Trans. on Signal Processing, Vo1. 40(5), pp.1273-1276.

Shentov, O.V., Hossen, A.N., Mitra, S.K. and U. Heute, 1991, "Sub-Band DFT-Interpretation, Accuracy, and Computational Complexity," Proc. of 25th Annual Asilomar Conf.Sig., Syst., Comp., Pacific Grove, CA, pp. 95100 .

Sorensen, H.V., Heideman, M.T. and Burrus, C.S., 1986, "On Computing the Split-Radix FFT," IEEE Trans. Acoust., Speech, Signal Processing, Vol. ASSP-34(1), pp. 152-156.

Sorensen, H.V., Burrus, C.S. and Jones, D..L., 1988, "A New Efficient Algorithm for Computing a Few DFT Points," Proc. of IEEE Int. Symp. on Circuits and Syst., Finland, pp. 1915-1918.

Sorensen, R.V. and Burrus, C.S., 1993, "Efficient Computation of the DFT with only a Subset of Input or Output Points," IEEE Trans. on Signal Processing, Vol. 41(3), pp. 1184-1199.

Sreenivas, T.V. and Rao, P.V.S., 1980, "High-Resolution Narrow-Band Spectra by FFT Pruning," IEEE Trans. Acoust., Speech, Signal Processing, Vol. ASSP-28(2), pp. 254-257.

Thrane, N., 1980, "Zoom FFT," Brüel \& Kjaer Technical Review, pp. 3-45.

Weeks, M., 2007, "Digital Signal Processing Using Matlab and Wavelets," Infinity Science Press.

Wild, R. de., Nieuwkerk, L.R. and Van Sinttruyen, J.S., 1987, "Method for Partial Spectrum Computation," IEE Proceedings, Vol. 134(7), Pt. F, pp. 659-666. 